\title{
MRI-Based Normative Measurement of the Ocular Globe Position in Relation to Inter-Zygomatic Line among Nepalese Subjects
}

Raj J B and Prajjwal U*

Department of Radiology and Imaging, TU Teaching

Hospital, Maharajgunj, Kathmandu, Nepal

*Corresponding author: Prajjwal Upadhayaya; Department of Radiology and Imaging; TU Teaching Hospital, Maharajgunj; Kathmandu, Nepal

Received: J uly 15, 2021; Accepted: August 12, 2021; Published: August 19, 2021

\begin{abstract}
Introduction: Proptosis and enophthalmos are cardinal signs of many orbito-ocular and systemic diseases. The need for an imaging parameter that will aid its early detection is necessary, as visual compromise is a major consequence if they are not diagnosed and managed early.

Methodology: This prospective study was performed in a tertiary center. Data were collected over the period of January to April 2021 with total of 300 normal ocular globes. The measurement was done at the level of lens (midglobe section) on T2-weighted axial image.

Results: The distance between the anterior margin and inter zygomatic line of the right ocular globe was $16.95 \pm 1.48 \mathrm{~mm}$ (ranged $14.2-20.5 \mathrm{~mm}$ ) and that of left was $16.86 \pm 1.38 \mathrm{~mm}$ (ranged $14.2-20.3 \mathrm{~mm}$ ). The position of the globes showed no statistically significant differences among gender groups in our study and the position of the right globe within the orbit was significantly different from that of the left orbit.

Conclusion: The position of the globes showed higher values in males than in female. However, a statistically significant difference in globe position was observed between right and left orbits. The distance between the posterior margin of the globe and the inter zygomatic line was found to be lower among the Nepalese population compared with other population.
\end{abstract}

Keywords: Interzygomatic line; Magnetic resonance imaging; Ocular globe; Proptosis

\section{Introduction}

The position of the ocular globe within the orbit is objectively assessed in relation to the Interzygomatic Line (IZL), which is a line joining the anterior margin of the zygomas [1,2]. The perpendicular distance of the anterior and posterior margins of the globe to the IZL at the level of the lens were recorded (Figure 1) [2]. Establishing a much-needed nomogram will help in making the early diagnosis of proptosis and enophthalmos. As there may be racial variations, there is a need for research to establish a nomogram, which will stand as a baseline for Nepalese subjects. The specific objectives were to measure the length of the IZL, distance between the anterior and posterior margins of the globes and IZL and to determine the variation of orbital measurements with age \& gender of individuals.

\section{Methodology}

This prospective study was performed in a tertiary center in Kathmandu. Data were collected over the period of January to April 2021 with total of 300 normal ocular globes. The measurement was done at the level of lens (mid-globe section) on T2-weighted axial image of 1.5T MRI scanner (Siemens Magnetom Amira). Purposive Non-Probability sampling method was used to select 150 patients. Inclusion criteria were patients referred for MRI scan of Brain/ PNS between the ages of 18 years and above but without clinical or radiological evidence of proptosis. Exclusion criteria were patients with endocrine diseases, which affect the orbit, particularly thyroid ophthalmopathy and those with orbital disorders; patients with asymmetric scans, scans with artifacts for any reason (e.g. eye implants, eye motion, etc.) that may cause errors in orbital measurements; patients with ferromagnetic prosthesis and Non-Nepalese patient.

The IZL is drawn between the most anterior part of zygomatic bone with electronic caliper and length was measured. It was used as a reference line. Distance between the anterior margin of the globe and IZL is measured by drawing the perpendicular line from IZL to the corneal apex on right and left globe to identify right and left anterior margin (RAM and LAM). Distance between the posterior margin of the globe and IZL was measured by drawing the perpendicular line from IZL to the posterior border on right and left globe to identify right and left posterior margin (RPM and LPM).

Data were analyzed using Statistical Package for Social Studies (SPSS, version 20) and Microsoft Excel 2013 software. The quantitative value of IZL, RAM, RPM, LAM and LPM is analyzed using ShapiroWilk test for normal distribution. For the sample collected for my study, the value of IZL, LPM and RPM were normally distributed and the value of RAM and LAM were not normally distributed. For normally distributed data, Paired samples t-test and Karl Pearson's Correlation Coefficient was used for statistical analysis and for data 
not normally distributed, Spearman Correlation Coefficient was used for analysis. A 95\% confidence interval was taken and p-value less than $0.05(\mathrm{p}<0.05)$ was taken as statistically significant.

\section{Results}

A total of 150 patients were enrolled in the study. As per gender wise distribution, 81 (54.0\%) patients comprised of female and 69 (46.0\%) patients were male. The mean age of the patients was $41.65 \pm$ 17.46 years with the minimum age being 18 years and the maximum age being 85 years. The maximum number of patients was between the age group of 20 to 49 years.

There were statistically significant differences found between data for the right and left orbits for all the measurements $(\mathrm{p}<0.05)$.

All the measurements showed higher values in males than in female. Statistically, there was no significant differences between male and female patients in all measurements $(\mathrm{p}>0.05)$ with the notable exception in the IZL which showed the statistically significant differences between males and females $(\mathrm{p}<0.05)$.

There was statistical significant correlation between age and right and left orbital parameters $(\mathrm{p}<0.05)$. A statistically weak negative correlation was found between age and RAM with the ' $r$ ' value of $-0.230(\mathrm{p}<0.05)$. A statistically weak positive correlation was found between age and RPM with the ' $r$ ' value of $0.180(\mathrm{p}<0.05)$ (Figure 2).

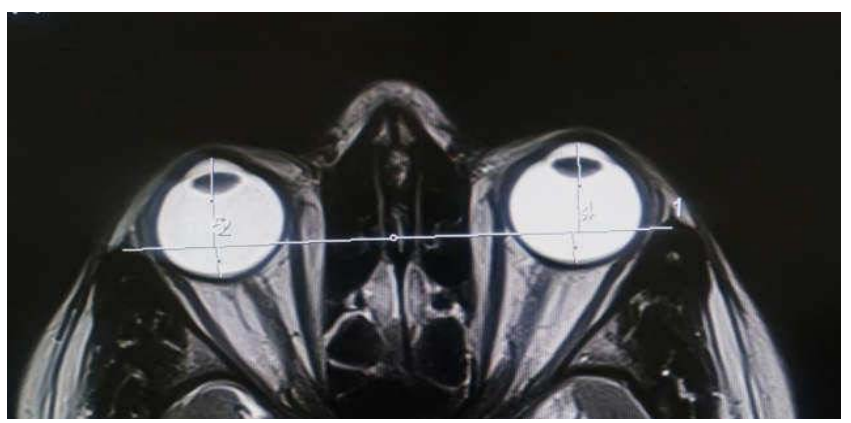

Figure 1: Measurement of ocular globe position on T2 weighted axial image of brain.

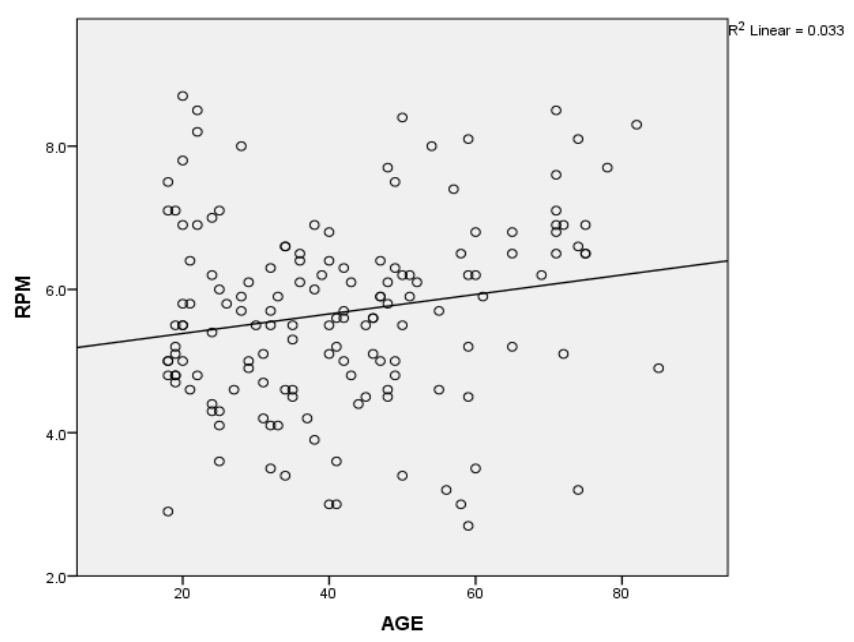

Figure 2: Linear correlation of Age with RPM showing weak positive correlation.
Table 1: Mean values, standard deviation and range of all the measurements in the study.

\begin{tabular}{|c|c|c|}
\hline Parameter & Mean \pm SD & Range \\
\hline IZL & $97.74 \pm 3.74 \mathrm{~mm}$ & $88.2-106.9 \mathrm{~mm}$ \\
\hline RAM & $16.95 \pm 1.48 \mathrm{Mm}$ & $14.2-20.5 \mathrm{~mm}$ \\
\hline RPM & $5.68 \pm 1.31 \mathrm{Mm}$ & $2.7-8.7 \mathrm{~mm}$ \\
\hline LAM & $16.86 \pm 1.38 \mathrm{Mm}$ & $14.2-20.3 \mathrm{~mm}$ \\
\hline LPM & $5.78 \pm 1.24 \mathrm{Mm}$ & $3.0-8.9 \mathrm{~mm}$ \\
\hline
\end{tabular}

Table 2: Gender-wise stratification of mean values and standard deviation of all the measurements.

\begin{tabular}{|c|c|c|c|c|}
\hline \multirow{2}{*}{ Parameters } & \multicolumn{2}{|c|}{ Mean SD } & Mean Difference & p-value \\
\cline { 2 - 5 } & Male (n=69) & Female (n=81) & & \\
\hline AGE & $42.91 \pm 18.42$ & $40.57 \pm 16.63$ & 2.34 & \\
\hline IZL (mm) & $98.82 \pm 3.81$ & $96.82 \pm 3.44$ & 2 & 0.001 \\
\hline RAM (mm) & $17.14 \pm 1.52$ & $16.79 \pm 1.43$ & 0.35 & 0.13 \\
\hline RPM (mm) & $5.71 \pm 1.38$ & $5.65 \pm 1.26$ & 0.06 & 0.782 \\
\hline LAM (mm) & $16.99 \pm 1.37$ & $16.74 \pm 1.39$ & 0.25 & 0.192 \\
\hline LPM (mm) & $5.88 \pm 1.28$ & $5.69 \pm 1.22$ & 0.19 & 0.347 \\
\hline
\end{tabular}

Similarly, a statistically weak negative correlation was found between age and LAM with the ' $r$ ' value of -0.219 ( $\mathrm{p}<0.05)$. A statistically weak positive correlation was found between age and LPM with the ' $r$ ' value of $0.165(\mathrm{p}<0.05)$.

\section{Discussion}

Proptosis and enopththalmos are cardinal signs of many orbitoocular and systemic diseases which are common in our environment. The need for an imaging parameter that will aid its early diagnosis is necessary, as visual compromise is a major consequence if they are not diagnosed and managed early. Although the CT offers rapid image acquisition and appears to be currently more widely available, CT exposes the patient to the ionizing radiation which may limits its routine utilization. In comparison, MRI offers very good contrast resolution of the soft tissues without exposing the patients to ionizing radiation and has multiplanar imaging capability. Although MRI is less available and needs longer image acquisition time as compared to $\mathrm{CT}, \mathrm{MRI}$ is considered as the key imaging modalities of choice for the soft tissue demonstration.

The mean length of the IZL was found to be significantly higher in males than in females. This observation is in tandem with the larger head size usually found in male [3] and it concurs with the finding of other authors. Aiyekomogbon [4], Ozgen and Aydingoz [5], Ozgen \& Ariyurek [6], Mohapatra et al. [7] and Lee et al. [8] found that IZL length was significantly higher among male participants. Gupta et al. [9] performed using CT scan in 2019 in India and found IZL was higher in males than in females.

Our study revealed a significant difference in the position of the right and left ocular globes within the orbits. Aiyekomogbon et al. [4] also found the similar findings but this finding is not consonance with the outcome of similar studies done by other authors in other parts of the world [5,7,10]. Ozgen and Aydingoz [5] did not find any significant statistical difference between data for the right and left orbits. There was also no significant difference in parameters for right 
Table 3: Mean values and Standard deviation of all parameters in different age groups in the study.

\begin{tabular}{|c|c|c|c|c|c|c|}
\hline \multirow{2}{*}{ Age groups } & \multirow{2}{*}{$n$} & \multicolumn{5}{|c|}{ Mean \pm SD } \\
\hline & & IZL (mm) & RAM (mm) & RPM (mm) & LAM (mm) & LPM (mm) \\
\hline $18-19$ & 13 & $96.53 \pm 3.17$ & $17.46 \pm 1.47$ & $5.34 \pm 1.23$ & $17.45 \pm 1.50$ & $5.43 \pm 1.19$ \\
\hline $20-29$ & 32 & $96.87 \pm 4.28$ & $16.85 \pm 1.25$ & $5.90 \pm 1.24$ & $16.79 \pm 1.24$ & $5.94 \pm 1.41$ \\
\hline $30-39$ & 27 & $97.44 \pm 4.43$ & $17.41 \pm 1.47$ & $5.25 \pm 1.04$ & $17.13 \pm 1.41$ & $5.49 \pm 0.97$ \\
\hline $40-49$ & 34 & $98.75 \pm 2.98$ & $17.17 \pm 1.48$ & $5.40 \pm 1.05$ & $17.08 \pm 1.35$ & $5.52 \pm 0.91$ \\
\hline $50-59$ & 19 & $97.42 \pm 3.85$ & $16.80 \pm 1.81$ & $5.62 \pm 1.73$ & $16.68 \pm 1.69$ & $5.73 \pm 1.63$ \\
\hline $60-69$ & 8 & $99.25 \pm 2.98$ & $16.56 \pm 0.93$ & $5.88 \pm 1.09$ & $16.58 \pm 1.03$ & $5.95 \pm 1.11$ \\
\hline$>70$ & 17 & $98.41 \pm 3.10$ & $15.94 \pm 1.30$ & $6.71 \pm 1.32$ & $15.95 \pm 1.00$ & $6.68 \pm 1.21$ \\
\hline
\end{tabular}

Table 4: Correlation of age with right and left orbital parameters.

\begin{tabular}{|c|c|c|}
\hline Parameters & Coefficient of Correlation $(\mathbf{r})$ & P-value \\
\hline RAM & -0.23 & 0.005 \\
\hline RPM & 0.18 & 0.027 \\
\hline LAM & -0.219 & 0.007 \\
\hline LPM & 0.165 & 0.043 \\
\hline
\end{tabular}

and left orbits in the studies of Lee et al. [5], Schmidt et al. [10] and that of Detorakis et al. [11]. The discordance observed in the outcome of this study and earlier research works enumerated above may be due to differences in technique and possibility of asymmetrical extraocular muscles contraction that could occur during the scan and the parameters that normally results in prolonged scan time as against MRI systems used by earlier authors. Furthermore, some of the authors used CT for their studies. Image acquisition is faster with $\mathrm{CT}$ and asymmetrical extraocular muscles contraction may not occur with this modality.

In our study, the posterior margin of the right ocular globe was $5.68 \pm 1.31 \mathrm{~mm}$ (ranged $2.7-8.7 \mathrm{~mm}$ ) behind the IZL while the left was $5.78 \pm 1.24 \mathrm{~mm}$ (ranged $3.0-8.9 \mathrm{~mm}$ ) from the IZL. Aiyekomogbon et al. [4] found that the posterior margin of the right ocular globe was $6.34 \pm 0.99 \mathrm{~mm}$ (ranged $5.4-7.3 \mathrm{~mm}$ ) behind the IZL while the left was $6.56 \pm 0.93 \mathrm{~mm}$ (ranged $5.6-7.5 \mathrm{~mm}$ ) from the IZL in Nigerian Population. Ozgen and Aydingoz [5] determined the normal position of the ocular globes with the aid of MRI in Turkey and discovered that the normal position of the posterior margin of the globe was $8.9 \mathrm{~mm}$ behind the IZL (ranged $5-12.7 \mathrm{~mm}$ ). Ozgen and Ariyurek [11] used CT for similar study in Turkey and found a mean value of $9.4 \mathrm{~mm}$ (ranged $5.9-12.8 \mathrm{~mm}$ ) as the distance between the IZL and posterior margin of the globes. Lee et al. [8] also carried out similar study among Koreans and found normal position of the ocular globe to be $11.2 \mathrm{~mm}$ (ranged $6.4-15.3 \mathrm{~mm}$ ) behind the IZL. A similar study in Canada by Nugent et al. [12] revealed that $95 \%$ of the cases had the posterior margins of their globes lying $6.5 \mathrm{~mm}$ or more behind the IZL. Gupta et al. [9] found that the normal position of the posterior pole from IZL was $7.9 \mathrm{~mm}$ and $7.8 \mathrm{~mm}$ for left and right sides, respectively among the Indian subjects using CT scan.

In our study, the distance between the anterior margin and IZL of the right ocular globe was $16.95 \pm 1.48 \mathrm{~mm}$ (ranged $14.2-20.5$ $\mathrm{mm}$ ) and that of left was $16.86 \pm 1.38 \mathrm{~mm}$ (ranged $14.2-20.3 \mathrm{~mm}$ ). Aiyekomogbon et al. [4] found that the distance between the right anterior margin and IZL was $17.01 \pm 1.56 \mathrm{~mm}$ while that of left anterior margin was $16.72 \pm 1.43 \mathrm{~mm}$ in Nigerian populations. Schmidt et al. [10] found that the distance between the anterior margin of globe and IZL to be $16.5 \pm 2.2 \mathrm{~mm}$ in men and that of women to be $15.3 \pm 2.1$ $\mathrm{mm}$ among the Northeast German adult population.

The differences that existed between the findings regarding the position of the ocular globes in the present study among Nepalese and findings among Nigerian, Turkish Koreans and others Populations as stated above might be due to racial variation and environmental influence. Benjamin [13] published an epidemiological study concerning ethnic differences, which suggested a general pattern of the highest prevalence of myopia in Asian, intermediate in Caucasian, and lowest in African-American. There is a paucity of data regarding this in Nepal, making comparison difficult.

In our study, the statistically significant differences in all right and left orbital parameters were found among the studied ages. These findings do not concur with that of Aiyekomogbon [4] and Lee [8] where no statistically significant differences in orbital measurements were found among the studied age groups.

The position of the globes showed no statistically significant differences among gender groups in our study, which is similar to findings among other authors $[4,5,7,8,11,13,14]$ where the gender differences were not statistically significant.

The sample size was not adequate to generalize the result and required the measurement with large sample size. The extent of a potential bias effect, if any exists, should be minimized by the larger number of sample taken. The patient group in our study was not a randomly selected sample. We measured patient's parameter manually and the value may not be consistent. Also, the use of 1.5 Tesla MRI systems may be our limitation as image acquisition was slow as compared to higher strength magnetic field MRI system and possibility of asymmetrical extraocular muscles contraction cannot be ruled out absolutely.

\section{Conclusion}

There was no significant difference between male and female patients in orbital measurements. However, a statistically significant difference in globe position was observed between right and left orbits. The distance between the posterior margin of the globe and the IZL was found to be lower among the Nepalese population compared with Caucasians, Koreans, Nigerian and Canadian. This further affirms that race and environmental influence have significant effects on the position of the ocular globes. 


\section{References}

1. Sabharwal KK, Chouhan AL and Jain S. CT evaluation of proptosis. Indian Journal of Radiology and Imaging. 2006; 16: 683

2. Munshi I. The Investigation of Proptosis-Clinical Medicine. Ophthalmology, University of the Witwatersrand. 2000

3. Hudson HL, Levin L and Feldon SE. Graves exophthalmos unrelated to extraocular muscle enlargement: superior rectus muscle inflammation may induce venous obstruction. Ophthalmology. 1991; 98: 1495-1499.

4. Aiyekomogbon JO, Chom ND, Hamidu AU, Rafindadi AL, Ibinaiye PO and Igashi JB. Normative measurements of the ocular globe position in relation to interzygomatic line, using magnetic resonance imaging among adults in Zaria, Nigeria. West African Journal of Radiology. 2016; 23: 118.

5. Özgen A and Aydingöz Ü. Normative measurements of orbital structures using MRI. Journal of computer assisted tomography. 2000; 24: 493-496.

6. Ozgen A and Ariyurek M. Normative measurements of orbital structures using CT. AJR. American journal of roentgenology. 1998; 170: 1093-1096.

7. Mohapatra M, Sahoo RK and Sahoo SR. Magnetic Resonance Imaging Evaluation of Normal Ocular Anterior-Posterior Diameter and Interzygomatic Length in Healthy Adult and Pediatric Eastern Indian Population: A Retrospective Study. Indian Journal of Public Health Research \& Development. 2019; 10.
8. Lee JS, Lim DW, Lee SH, Oum BS, Kim HJ and Lee HJ. Normative measurements of Korean orbital structures revealed by computerized tomography. Acta ophthalmologica Scandinavica. 2001; 79: 197-200.

9. Gupta V, Prabhakar A, Yadav M and Khandelwal N. Computed tomography imaging-based normative orbital measurement in Indian population. Indian journal of ophthalmology. 2019; 67: 659.

10. Schmidt P, Kempin R, Langner S, Beule A, Kindler S, Koppe T, et al. Association of anthropometric markers with globe position: A populationbased MRI study. PLoS One. 2019; 14.

11. Detorakis ET, Drakonaki EE, Papadaki E, Tsilimbaris MK and Pallikaris IG. Evaluation of globe position within the orbit: clinical and imaging correlations. British Journal of Ophthalmology. 2010; 94: 135-136.

12. Nugent RA, Belkin RI, Neigel JM, Rootman J, Robertson WD, Spinelli J, et al. Graves orbitopathy: correlation of CT and clinical findings. Radiology. 1990 177: 675-682.

13. Benjamin WJ. Borish's Clinical Refraction-E-Book. Elsevier Health Sciences. 2006.

14. Kirsch E and Hammer B. Graves' orbitopathy: current imaging procedures. Swiss medical weekly. 2009; 139. 